\title{
Erratum: Observation of ultrahigh mobility surface states in a topological crystalline insulator by infrared spectroscopy
}

Ying Wang ${ }^{1}$, Guoyu Luo ${ }^{2}$, Junwei Liü ${ }^{3,4}$, R. Sankar ${ }^{5,6}$, Nan-Lin Wang ${ }^{7}$, Fangcheng Chou ${ }^{5}$, Liang Fu ${ }^{3}$ \& Zhiqiang Li

Nature Communications 8:366 10.1038/s41467-017-00446-2; Article published online: 28 August 2017

This Article contains two typesetting errors. In the penultimate sentence of the first paragraph of the Results section, the equation should be ' $\alpha_{0}(\omega) \propto \sqrt{\hbar \omega-\Delta / \hbar \omega}$ ', not ' $\alpha_{0}(\omega) \propto \sqrt{\hbar \omega-\Delta / \hbar \omega}$ '. In Fig. 4a, the variable marking the top dashed line should be ' $E_{\mathrm{H} 1}^{\mathrm{DP}}$, not ' $E_{\mathrm{H} 2}^{\mathrm{DP}}$ '.

Published online: 12 October 2017

\begin{abstract}
(c) (i) Open Access This article is licensed under a Creative Commons Attribution 4.0 International License, which permits use, sharing, adaptation, distribution and reproduction in any medium or format, as long as you give appropriate credit to the original author(s) and the source, provide a link to the Creative Commons license, and indicate if changes were made. The images or other third party material in this article are included in the article's Creative Commons license, unless indicated otherwise in a credit line to the material. If material is not included in the article's Creative Commons license and your intended use is not permitted by statutory regulation or exceeds the permitted use, you will need to obtain permission directly from the copyright holder. To view a copy of this license, visit http://creativecommons.org/licenses/by/4.0/.
\end{abstract}

(๑) The Author(s) 2017

\footnotetext{
${ }^{1}$ National High Magnetic Field Laboratory, Tallahassee, FL 32310, USA. ${ }^{2}$ College of Physical Science and Technology, Sichuan University, Chengdu, Sichuan 610064, China. ${ }^{3}$ Department of Physics, Massachusetts Institute of Technology, Cambridge, MA 02139, USA. ${ }^{4}$ Department of Physics, Hong Kong University of Science and Technology, Clear Water Bay, Hong Kong, China. ${ }^{5}$ Center for Condensed Matter Sciences, National Taiwan University, Taipei, 10617, Taiwan. ${ }^{6}$ Institute of Physics, Academia Sinica, Taipei 11529, Taiwan. ${ }^{7}$ International Center for Quantum Materials, School of Physics, Peking University, Beijing 100871, China. Correspondence and requests for materials should be addressed to Z.L. (email: zhiqiangli@scu.edu.cn)
} 